\title{
Vegetación leñosa de un remanente de bosque seco tropical en el Caribe colombiano
}

\section{Woody vegetation of a tropical dry forest remnant in the Colombian Caribbean}

\author{
Viviana Londoño-Lemos ${ }^{1 *(\mathbb{1}}$.Juan Tapasco-García ${ }^{\circledR}{ }^{(}$ \\ Orlando Escobar-Hadechini ${ }^{\oplus}$, Santiago Madriñán ${ }^{\oplus}$
}

Londoño-Lemos, V., Tapasco-García, J., Escobar-Hadechini, O. y Madriñán, S. (2022). Vegetación leñosa de un remanente de bosque seco tropical en el Caribe colombiano. Colombia Forestal, 25(1), 51-66.

Recepción: 26 de abril 2021

\section{Resumen}

El bosque seco tropical del Jardín Botánico de Cartagena es uno de los pocos remanentes protegidos cerca de esta ciudad. Su estado de conservación y la abundancia de nacimientos de agua en la zona lo hacen único entre los remanentes de este ecosistema en el Caribe colombiano. En este contexto, el objetivo del presente estudio fue evaluar la estructura, composición, diversidad funcional y filogenética de la comunidad vegetal del área mediante una parcela permanente. Para ello se registraron 2023 tallos de 62 especies y la familia con mayor Índice de Valor de Importancia (IVI) fue Moraceae. La biomasa aérea total fue de 61.8 t.ha $^{-1}$. Se encontraron dos tipos funcionales de plantas: uno adaptado al sotobosque y otro al dosel, y un bajo agrupamiento filogenético. Se concluyó que el bosque se encuentra en un estado de sucesión secundario intermedio a tardío y que la alta disponibilidad de agua en la zona, además de su ubicación aislada, han sido factores determinantes para su vegetación.

Palabras clave: biomasa, composición florística, diversidad filogenética, diversidad funcional, estructura vegetal, rasgos funcionales.
Aprobación: 18 de agosto 2021

\begin{abstract}
The Cartagena Botanical Garden tropical dry forest is one of the few protected forest remnants near Cartagena. Its conservation state and the abundance of water sources in the area make it unique among this ecosystem's remnants in the Colombian Caribbean. We evaluated the structure, composition, and the functional and phylogenetic diversity of the plant community by setting up a permanent plot. We recorded 2023 ramets of 62 species. The family with the highest Importance Value Index (IVI) was Moraceae. The total aboveground biomass was 61.8 t.ha $^{-1}$. Two functional types of plants were found: one adapted to the understory, and the other one adapted to the canopy, and a low phylogenetic grouping. We concluded that the forest is in a late to intermediate secondary succession state. The high availability of water in the area and its isolated location have been determining factors for its vegetation.
\end{abstract}

Keywords: biomass, floristic composition, phylogenetic diversity, functional diversity, plant structure, functional traits.

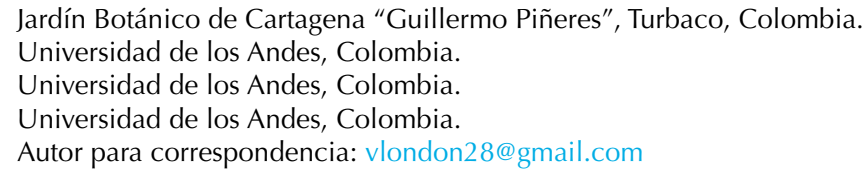




\section{INTRODUCCIÓN}

El bosque seco tropical (bs-T) es un bioma que agrupa diversos tipos de formaciones vegetales que van desde matorrales xéricos hasta bosques siempreverdes y caducifolios (Pennington et al., 2009). El bs-T se caracteriza por presentar una marcada estacionalidad, con 3 a 8 meses de sequía, una o dos épocas de lluvia al año y un rango de precipitación anual entre 250 y 2000 mm (Gentry, 1995; Murphy y Lugo, 1986). Esta estacionalidad supone la aparición de adaptaciones especiales de las plantas como respuesta al déficit hídrico, lo que significa altas tasas de endemismo y diversidad beta (DRYFLOR et al., 20 16; Pizano et al., 2014). La mayoría de los bs-T son estructuralmente menos complejos que los bosques húmedos tropicales, presentando menores valores de altura, área basal, biomasa y riqueza de especies; esto puede deberse a factores como el estado de conservación de los bosques, el clima y el tipo de suelo (Maia et al., 2020; Murphy y Lugo, 1986), y a la presencia de un sotobosque con mayor densidad de arbustos y abundancia de lianas (Gentry, 1995). En cuanto a su composición, el bs-T se caracteriza por la dominancia general de Fabaceae en el estrato arbóreo y de Bignoniaceae para las lianas (Gentry, 1995; Murphy y Lugo, 1986).

A nivel mundial Sudamérica concentra el $54 \%$ de los bs-T (Miles et al., 2006) y, en particular, para el caso de Colombia, son seis las agrupaciones de bs-T que se presentan de acuerdo con la variabilidad ambiental y la composición florística (González-M et al., 2018), de la cuales la región Caribe es la que contiene los fragmentos de mayor extensión (DRYFLOR et al., 2016; Pizano et al., 2016). Actualmente, menos del $8 \%$ del tamaño original de bs-T en Colombia se mantiene, y sólo el $5 \%$ del área remanente se encuentra protegida (Pizano et al., 2014). Los ecosistemas secos se encuentran entre los más vulnerables a nivel global y, en los trópicos, el bs-T es de los más amenazados (FAO, 2019; Miles et al., 2006;
Pizano et al., 2014; Sánchez-Azofeifa et al., 2003). En este contexto, durante la última década diversas investigaciones han evaluado el estado de comunidades vegetales de bs-T en distintas etapas de sucesión en el país (DRYFLOR et al., 2016; Pizano et al., 2014; Polania Cleves, 2019; Ruiz Linares y Fandiño Orozco, 2009), pero a pesar de estas contribuciones varios estudios han señalado la importancia de continuar generando información sobre el bs-T en Colombia e implementando estudios a largo plazo, particularmente en la región Caribe (Álvarez et al., 2012; Miles et al., 2006).

Entre los remanentes de bs-T cercanos a la ciudad de Cartagena, una de las más importantes y con mayor desarrollo urbano del Caribe colombiano, se encuentra el bosque que protege el Jardín Botánico "Guillermo Piñeres" (JBGP). Este remanente aislado está rodeado por una matriz rural intervenida, alejado por lo menos $5 \mathrm{~km}$ a la redonda de otros fragmentos de tamaño similar (entre 1 a 10 ha). Es debido al estado de fragmentación del bs-T en esta zona que esta área se ha convertido en un refugio para la fauna y flora nativas. Otra de sus características únicas es que, pese a que presenta la estacionalidad usual del ecosistema, la disponibilidad de agua es alta a lo largo del año, pues existen varios acuíferos y nacimientos pertenecientes a la cuenca del arroyo Matute, uno de los afluentes más importantes de Cartagena (Botero Pareja et al., 2008). Cabe señalar que el fragmento no ha sido intervenido desde 1972 cuando se construyó un sendero peatonal, por lo cual su vegetación cuenta con casi 50 años de recuperación.

Por todo lo anterior, en este estudio se evaluó la comunidad vegetal leñosa del bs-T perteneciente al JBGP mediante la determinación de su estructura, composición florística y diversidad funcional y filogenética. Además, se sentó una base para su monitoreo gracias a la construcción de una parcela permanente de 1 ha con el objetivo de continuar generando conocimientos sobre la vegetación de los fragmentos aislados de bs-T en matrices altamente intervenidas. 


\section{MATERIALES Y MÉTODOS}

\section{Área de estudio}

El JBGP $\left(10^{\circ} 21^{\prime} 16^{\prime \prime} \mathrm{N}, 75^{\circ} 25^{\prime} 40^{\prime \prime} \mathrm{W}\right)$ se localiza a $5 \mathrm{~km}$ de Cartagena y está bajo la jurisdicción del municipio de Turbaco (Bolívar, Colombia). El remanente de bs-T que protege cuenta con una extensión de 3 ha, su altitud oscila entre los 167 m y $190 \mathrm{~m}$, y la zona recibe en promedio $1238 \mathrm{~mm}$ de precipitación anuales, con una estación seca que va desde diciembre hasta marzo y en la que caen menos de $50 \mathrm{~mm}$ mensuales. La temperatura promedio anual es de $28{ }^{\circ} \mathrm{C}$ (mediciones del JBGP).

\section{Establecimiento y censo de la parcela}

La parcela permanente fue construida y censada siguiendo los protocolos descritos en Vallejo-Joyas et al. (2005); esta constaba de 1 ha dividida en 25 cuadrantes de 20 m x 20 m. La delimitación de los cuadrantes y de la parcela fue realizada con un hipsómetro TruPulse $360 \mathrm{R}$ de Laser Technology. Se marcaron todos los tallos de individuos leñosos con diámetro a la altura del pecho (DAP) mayor o igual a $2.5 \mathrm{~cm}$ y a cada tallo le fue asignada una placa con un código alfanumérico para su posterior censo, ubicada a una altura de $1.8 \mathrm{~m}$.

De cada uno de los tallos censados se registró la especie, el DAP, la altura total y su ubicación midiendo con un decámetro la distancia a los ejes de cada cuadrante (con precisión de $10 \mathrm{~cm}$ ). Además, se reunieron muestras de cada especie para su identificación, depositadas en el herbario del JBGP con el nombre del proyecto: "Parcela Permanente JBGP 2020" y colectadas bajo el permiso del Marco de Recolección de la Resolución No. 000571 de 2015.

\section{Composición y estructura florística}

Se calcularon los índices de diversidad de Shannon $\left(\mathrm{H}^{\prime}\right)$ y la exponencial de Shannon $\left(\mathrm{e}^{\mathrm{H}}\right)$ y Simpson (1 - D) (Jost, 2007). Así mismo, se realizó un análisis de similitud ANOSIM para saber si existían diferencias de composición florística entre los cuadrantes de la parcela. Estos análisis fueron realizados con el software PAST, versión 4.0.2. (Hammer et al., 2001). En cuanto al estudio de la estructura del bosque, se calculó el área basal y se determinaron los valores de frecuencia, abundancia y dominancia relativas para el cálculo del índice de valor de importancia (IVI) por especie.

\section{Diversidad funcional}

Se seleccionaron las especies cuya abundancia relativa acumulada en la parcela sumara aproximadamente el 80 \% para la medición de los rasgos funcionales. De cada especie se escogieron 10 individuos de altura superior a la media por especie y de cada uno se colectaron 6 hojas que estuvieran expuestas al sol (Pérez-Harguindeguy et al., 2013). A continuación, se midieron 4 tipos de rasgos: generales, foliares, del tallo y regenerativos (Tabla 1). Luego, a partir de los datos de rasgos por especie, se calcularon los índices de diversidad funcional, utilizando la media ponderada por comunidad y estandarizando los datos para equilibrar sus varianzas. Cabe mencionar que el análisis de la diversidad funcional se realizó en el programa FDiversity, versión 2014 (Casanoves et al., 2010). Los Índices calculados fueron el rRao (rao relativo), como un medidor relativo de la diversidad general, y los índices funcionales Fric (riqueza funcional, el espacio funcional ocupado por la comunidad), Feve (igualdad funcional, la regularidad de la distribución de las abundancias de las especies en el espacio funcional), Fdiv (divergencia funcional, la divergencia de las abundancias de las especies al centroide del espacio funcional), Fdis (dispersión funcional, distancia promedio de cada especie y sus rasgos al centroide) y Fspe (especialización funcional, el promedio de la especialización de las especies dentro de la comunidad) (Casanoves et al., 2010; Casanoves et al., 2011; Villéger et al., 2010). 
Posteriormente, se llevó a cabo un dendrograma con el método de análisis jerárquico de Ward y distancias euclidianas para caracterizar los tipos funcionales de las especies más importantes. En seguida, se aplicó un análisis PERMANOVA, debido a que los datos no se distribuían normalmente, para evaluar la significancia de la diferencia entre estos grupos funcionales. Y, finalmente, se realizó un análisis de componentes principales (PCA) para observar la separación entre los grupos y los caracteres que mayormente incidían en la separación observada.

\section{Biomasa}

Los valores de densidad de madera para el cálculo de biomasa se obtuvieron del Global Wood Density Database (Zanne et al., 2009), a excepción de Sabal mauritiiformis, cuyo valor se obtuvo de Álvarez et al. (2013). En el caso de especies de lianas de las que no se encontró información, se adoptó un valor estándar de 0.4 g. $\mathrm{cm}^{-3}$ (Putz, 1990). Utilizando dos ecuaciones alométricas, se calculó la biomasa aérea para lianas (Schnitzer et al., 2006) y árboles (Chave et al., 2014). Se escogieron estas ecuaciones dada su robustez a lo largo de distintos ecosistemas boscosos y condiciones bioclimáticas, y en el caso de las lianas, debido a su aplicabilidad a los diversos tipos de lianas encontrados en el bosque.

\section{Diversidad filogenética}

Se construyó una filogenia de todas las especies de la parcela utilizando el programa web Phylomatic 3.0, basado en la base de datos del sistema de clasificación APG III (Webb y Donoghue, 2005). El árbol resultante fue convertido a ultramétrico, donde todas las ramas tienen la misma distancia al origen y se asume la presencia de reloj molecular evolutivo, lo que permite calcular con mayor facilidad las distancias filogenéticas de las especies en la comunidad. Se utilizó, además, el programa FDiversity para calcular los índices PSV (phylogenetic species variability o variabilidad filogenética, definida como la cercanía filogenética entre las especies), PSR

Tabla 1. Tipos de rasgos funcionales medidos para las especies que representan el $80 \%$ de la abundancia relativa acumulada

\begin{tabular}{|c|c|c|c|}
\hline Tipo de rasgo & Rasgo & Descripción & Metodología \\
\hline General & Altura máxima (m) & $\begin{array}{l}\text { Altura total del individuo de } \\
\text { mayor altura por especie }\end{array}$ & Datos del censo de la vegetación \\
\hline \multirow{2}{*}{ Foliar } & Área foliar $\left(\mathrm{cm}^{2}\right)$ & Área de la hoja y el peciolo & $\begin{array}{l}\text { Colecta de } 6 \text { hojas por individuo en } 10 \text { individuos, } \\
\text { área medida en software ImageJ (Abràmoff et al., } \\
\text { 2004) }\end{array}$ \\
\hline & $\begin{array}{l}\text { Área foliar específica } \\
\left(\mathrm{cm}^{2} \cdot \mathrm{g}^{-1}\right)\end{array}$ & $\begin{array}{l}\text { Relación entre área foliar y } \\
\text { masa seca }\end{array}$ & $\begin{array}{l}\text { La masa seca corresponde al peso de las hojas des- } \\
\text { pués de permananecer durante } 7 \text { días en un horno a } \\
60{ }^{\circ} \mathrm{C}\end{array}$ \\
\hline Tallo & Densidad de madera & $\begin{array}{l}\text { Relación entre la masa seca } \\
\text { y el volumen fresco de la } \\
\text { madera }\left(\mathrm{g} \cdot \mathrm{cm}^{-3}\right)\end{array}$ & $\begin{array}{l}\text { Consulta de fuentes bibliográficas: todas las de Zanne } \\
\text { et al., 2009, excepto Sabal mauritiiformis, analizada } \\
\text { por Álvarez et al. (2013) }\end{array}$ \\
\hline \multirow[b]{2}{*}{ Regenerativos } & Semillas por fruto & Número de semillas por fruto & $\begin{array}{l}\text { Promedio de número de semillas por fruto depen- } \\
\text { diendo de la disponibilidad de individuos y fuentes } \\
\text { bibliográficas }\end{array}$ \\
\hline & Masa de semillas & Peso seco de las semillas (g) & $\begin{array}{l}\text { Peso de las semillas después del secado en horno. } \\
\text { Las semillas grandes fueron trituradas. Para algunas } \\
\text { especies se consultaron las siguientes fuentes biblio- } \\
\text { gráficas: Cedeño et al., 2010; Faicán Ortiz e Intriago } \\
\text { Méndez, 2019; Francis y Rodríguez, 1993; Gerhardt, } \\
\text { 1993; Royal Botanical Gardens Kew, 2021 }\end{array}$ \\
\hline
\end{tabular}


(phylogenetic species richness, entendida como el número de especies o "grupos" de la comunidad teniendo en cuenta su cercanía filogenética), PSE (phylogenetic species eveness, que es la variabilidad de las especies -al igual que el PSV-, pero este índice considera la abundancia de las especies) y PSC (phylogenetic species clustering o agrupamiento filogenético de especies, una métrica que precisa cómo se agrupan las puntas de las ramas del árbol filogenético) (Helmus et al., 2007).

\section{RESULTADOS}

\section{Composición y estructura}

Los datos de las mediciones de la parcela pueden ser consultados a través de los portales de SiB Colombia y GBIF (Londoño-Lemos et al., 2020). Allí, fueron registrados 2023 tallos agrupados en 1602 individuos pertenecientes a 62 especies distribuidas en 34 familias (Anexo 1). El $85 \%$ de los individuos fueron árboles y arbustos, el $11 \%$ lianas y el $4 \%$ palmas. Las especies más abundantes, tanto en número de individuos como de tallos, fueron $\mathrm{Bro}-$ simum alicastrum (14\% y $17 \%$, respectivamente), seguida de Trophis racemosa (13\% y $17 \%$ ) y Capparidastrum frondosum (9\% y $11 \%$ ). Urera baccifera ocupó el cuarto lugar en número de tallos (9 \%) y Mammea americana ocupó el cuarto lugar en número de individuos (8\%). En cuanto a los índices de diversidad $\mathrm{H}^{\prime}$ y $\mathrm{e}^{\left(\mathrm{H}^{\prime}\right)}$, estos sugieren una dominancia por parte de las especies más importantes del bosque con sus respectivos valores de 2.884 y 17.886 . Por su parte, el índice de Simpson tuvo un valor de 0.911. La familia con mayor riqueza de especies fue Sapindaceae, con cuatro, seguida de Apocynaceae, Araceae, Fabaceae, Meliaceae, Moraceae, Nyctaginaceae y Urticaceae, cada una con tres. Para el resto de las familias (12) se registraron 1 o 2 especies. El ANOSIM no sugirió diferencias significativas entre los cuadrantes de las parcelas ( $p=0.0565, R=0.0291)$, por lo que las especies dentro del bosque tienen una distribución aleatoria dentro de la parcela. Respecto al área basal total para la parcela, esta fue de $20.9 \mathrm{~m}^{2}$.ha y las especies que más aportaron fueron Guarea guidonia (21\%), Ficus maxima (13\%), Trophis racemosa (12\%) y Sabal mauritiiformis (10\%). El aporte de las lianas al área basal fue de $4.01 \mathrm{~m}^{2}$.ha y las familias con mayor IVI acumulado fueron Moraceae, Capparaceae y Meliaceae, respectivamente. A nivel de especie, las que presentaron mayor IVI fueron Trophis racemosa (109\%, Moraceae) y Brosimum alicastrum (94\%, Moraceae), seguidas de Capparidastrum frondosum (70\%, Capparaceae) y Guarea guidonia (60\%, Meliaceae). Para el caso de las lianas se registraron 13 especies, de las cuales la más abundante fue la Bignoniaceae Martinella obovata (42\% IVI, 100 individuos). Es preciso señalar que todas las especies de Bignoniaceae registradas fueron lianas y que el resto de las especies de lianas no contó con un IVI significativo, dada su distribución localizada (Anexo 1).

\section{Biomasa}

Todos los cálculos de biomasa se realizaron con un n de 2023 tallos, dando como resultado una biomasa aérea total de 61.8 t.ha $^{-1}$. Las especies con mayor aporte de biomasa aérea fueron Guarea guidonia (25\%), Ficus maxima (12\%), Trophis racemosa (11\%) y Sabal mauritiiformis (8\%). Al analizar la distribución de la biomasa aérea entre las clases diamétricas, los individuos con DAP mayor a $30 \mathrm{~cm}$ concentraron el $55 \%$ (31.0 t.ha- ${ }^{1}$ ). En cuanto a la distribución de la biomasa aérea por altura, los tallos con altura superior a los $14 \mathrm{~m}$ concentraron el $61 \%$. Así mismo, las lianas aportaron 6.6 t.ha $^{-1}$ a la biomasa aérea total.

\section{Diversidad funcional}

Las especies que sumaron el $80 \%$ (12) de la abundancia relativa acumulada junto con los valores de cada rasgo por especie se resumen en la Tabla 2. Para calcular cada índice se utilizaron 6 hojas de 
10 individuos de cada especie, exceptuando la palma Sabal mauritiiformis para la cual, debido a la dificultad de colecta de sus hojas, los datos corresponden a 2 hojas por individuo en 5 individuos. Respecto a los datos no foliares, se utilizó la información recolectada a través de la bibliografía. Los siguientes fueron los valores obtenidos para los índices de diversidad funcional: $r$ Rao $=$ 0.4148, FRic $=0.17011$, FEve $=0.5628$, FDiv $=$ 0.7052 , FDis $=1.798$ y $\mathrm{FSpe}=2.2272$.

Con base al dendrograma obtenido se aprecian dos grupos funcionales distintos que presentan diferencias significativas según el PERMANOVA $(p=0.0018)$. Debido a que al excluir las especies con hábitos de crecimiento disímil (palma Sabal mauritiiformis y liana Martinella ovobata) las diferencias significativas se mantienen $(p=0.0033$, $\mathrm{n}=12$ ), se utilizaron todas las especies seleccionadas para los análisis. En este sentido, se obtuvo un grupo de especies arbóreas y una palma conformado por Trophis racemosa, Brosimum alicastrum, Guarea guidonia, Capparidastrum frondosum, Manilkara zapota, Manilkara chicle, Mammea americana y Sabal mauritiiformis, caracterizadas por tener una altura máxima que supera el dosel, alta densidad de madera y baja proporción de área específica foliar (Figura 1 y Figura 2). En contraste, el otro grupo se compone de arbustos y una liana, conformado por las especies Tabernaemontana amygdalifolia, Piper amalago, Urera baccifera y Martinella ovobata, que se identifican por tener baja densidad de madera, bajos valores de altura máxima, muy alta

Tabla 2. Rasgos funcionales evaluados de las 12 especies que representaron el $80 \%$ de la abundancia relativa acumulada, donde: $\mathrm{AM}=$ Altura máxima, $\mathrm{AF}=$ Área foliar, $\mathrm{AFE}=$ Área foliar específica, $\mathrm{D}=$ densidad. Los valores medidos representan promedios y se indica la desviación estándar

\begin{tabular}{|c|c|c|c|c|c|c|}
\hline \multirow[b]{3}{*}{ Especie } & \multicolumn{6}{|c|}{ Tipo de rasgo } \\
\hline & \multirow{2}{*}{$\begin{array}{l}\text { General } \\
\text { AM (m) }\end{array}$} & \multicolumn{2}{|l|}{ Foliar } & Tallo & \multicolumn{2}{|c|}{ Regenerativos } \\
\hline & & $\operatorname{AF}\left(\mathrm{cm}^{2}\right)$ & $\begin{array}{l}\text { AFE } \\
\left(\mathrm{cm}^{2} \cdot \mathrm{g}^{-1}\right)\end{array}$ & $D\left(\right.$ g. $\left.\mathrm{cm}^{-3}\right)$ & $\begin{array}{l}\text { Semillas } \\
\text { por fruto }\end{array}$ & $\begin{array}{l}\text { Masa semillas } \\
(\mathrm{g})\end{array}$ \\
\hline Brosimum alicastrum & 14.5 & $52.4 \pm 25.2$ & $16.8 \pm 6.5$ & $0.599 \pm 0.109$ & $1 \pm 0$ & $1.000 \pm 0.3$ \\
\hline $\begin{array}{l}\text { Capparidastrum frondo- } \\
\text { sum+ }\end{array}$ & 6.9 & $73.6 \pm-35.6$ & $11.7 \pm 1.8$ & $0.691 \pm 0.094$ & $7 \pm 3$ & $0.097 \pm 0.00$ \\
\hline Guarea guidonia+ & 24.0 & $1682.5 \pm 986.3$ & $13.2 \pm 6.5$ & $0.543 \pm 0.091$ & $3 \pm 1$ & $0.198 \pm 0.00$ \\
\hline Mammea americana+ & 20.3 & $102.7 \pm 31.6$ & $7.9 \pm 1.6$ & $0.64 \pm 0.028$ & $2 \pm 1$ & $44.000 \pm 13.68$ \\
\hline Manilkara chicle+ & 10.1 & $152.6 \pm 44.3$ & $11.4 \pm 3.5$ & $1.04 \pm 0.000$ & $6 \pm 2$ & $0.290 \pm 0.07$ \\
\hline Manilkara zapota & 14.1 & $33.3 \pm 15.4$ & $9.2 \pm 1.1$ & $0.81 \pm 0.000$ & $6 \pm 1$ & $0.216 \pm 0.001$ \\
\hline Martinella obovata & NA & $115.9 \pm 74.0$ & $28.1 \pm 22.4$ & $0.578 \pm 0.032$ & $55 \pm 27$ & $0.047 \pm 0.001$ \\
\hline Piper amalago+ & 5.9 & $55.8 \pm 14.4$ & $29.3 \pm 6.9$ & $0.394 \pm 0.089$ & $1 \pm 0$ & $0.001 \pm 0.002$ \\
\hline Sabal mauritiiformis* & 18.5 & $35296.2 \pm 5642.0$ & $3.7 \pm 1.1$ & $0.497 \pm 0.027$ & $1 \pm 0$ & $0.300 \pm 0.001$ \\
\hline $\begin{array}{l}\text { Tabernaemontana amygda- } \\
\text { lifolia }\end{array}$ & 5.6 & $36.1 \pm 16.3$ & $23.6 \pm 5.4$ & $0.524 \pm 0.103$ & $12 \pm 9$ & $0.027 \pm 0.001$ \\
\hline Trophis racemosa & 19.7 & $48.1 \pm 21.1$ & $16.5 \pm 4.4$ & $0.659 \pm 0.111$ & $1 \pm 0$ & $0.500 \pm 0.001$ \\
\hline Urera baccifera+ & 5.0 & $289.7 \pm 92.6$ & $31.4 \pm 11.0$ & $0.165 \pm 0.000$ & $1 \pm 0$ & $0.07 \pm 0.02$ \\
\hline
\end{tabular}

Nota: * Los valores de Sabal mauritiiformis para AFE y AF corresponden a muestras de dos hojas por individuo en cinco individuos; esto se debe a la dificultad de colectar una hoja completa sin causar daño. El valor de densidad fue obtenido de Álvarez et al. (2013).

+ Datos de semillas obtenidos de fuentes bibliográficas. 


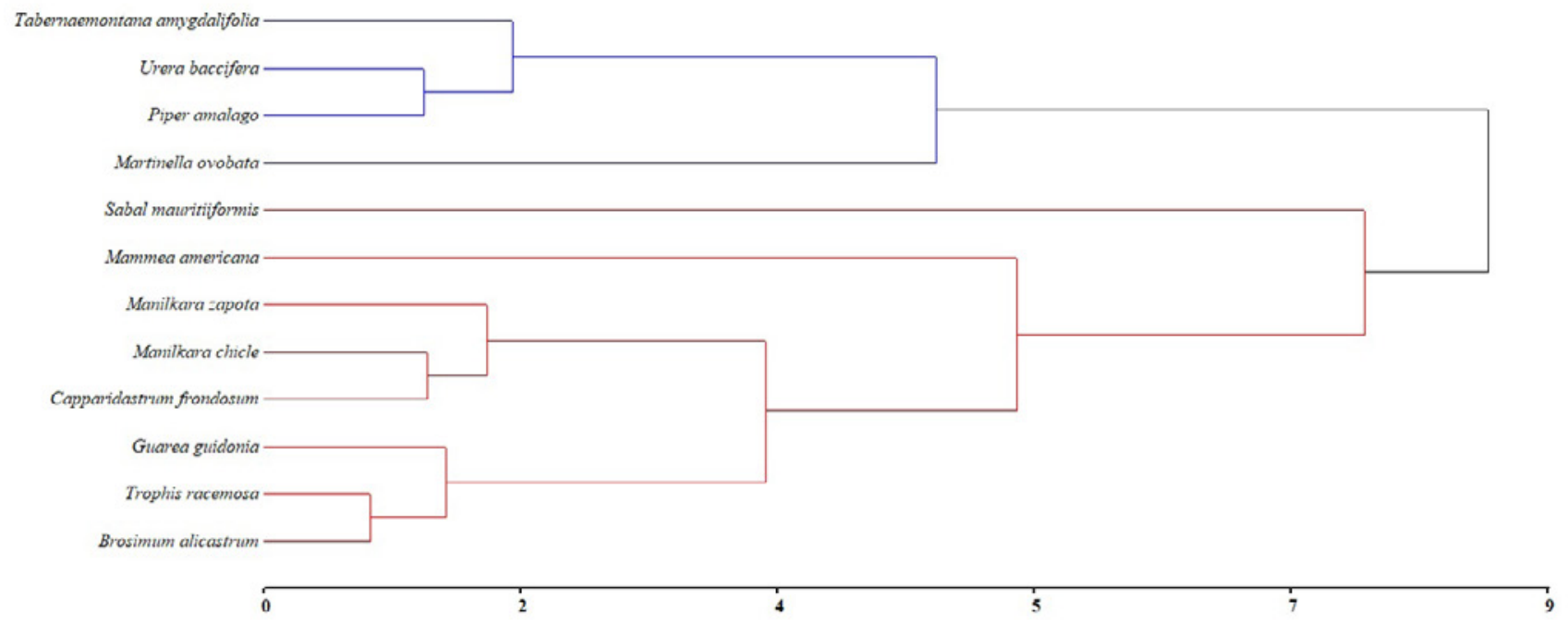

Figura 1. Dendrograma creado con el método de Ward y distancias euclidianas, basado en las especies que representan el $80 \%$ de la abundancia relativa acumulada de la parcela.

Se observan dos grupos funcionales: árboles/palma (rojo), arbusto/liana (azul).

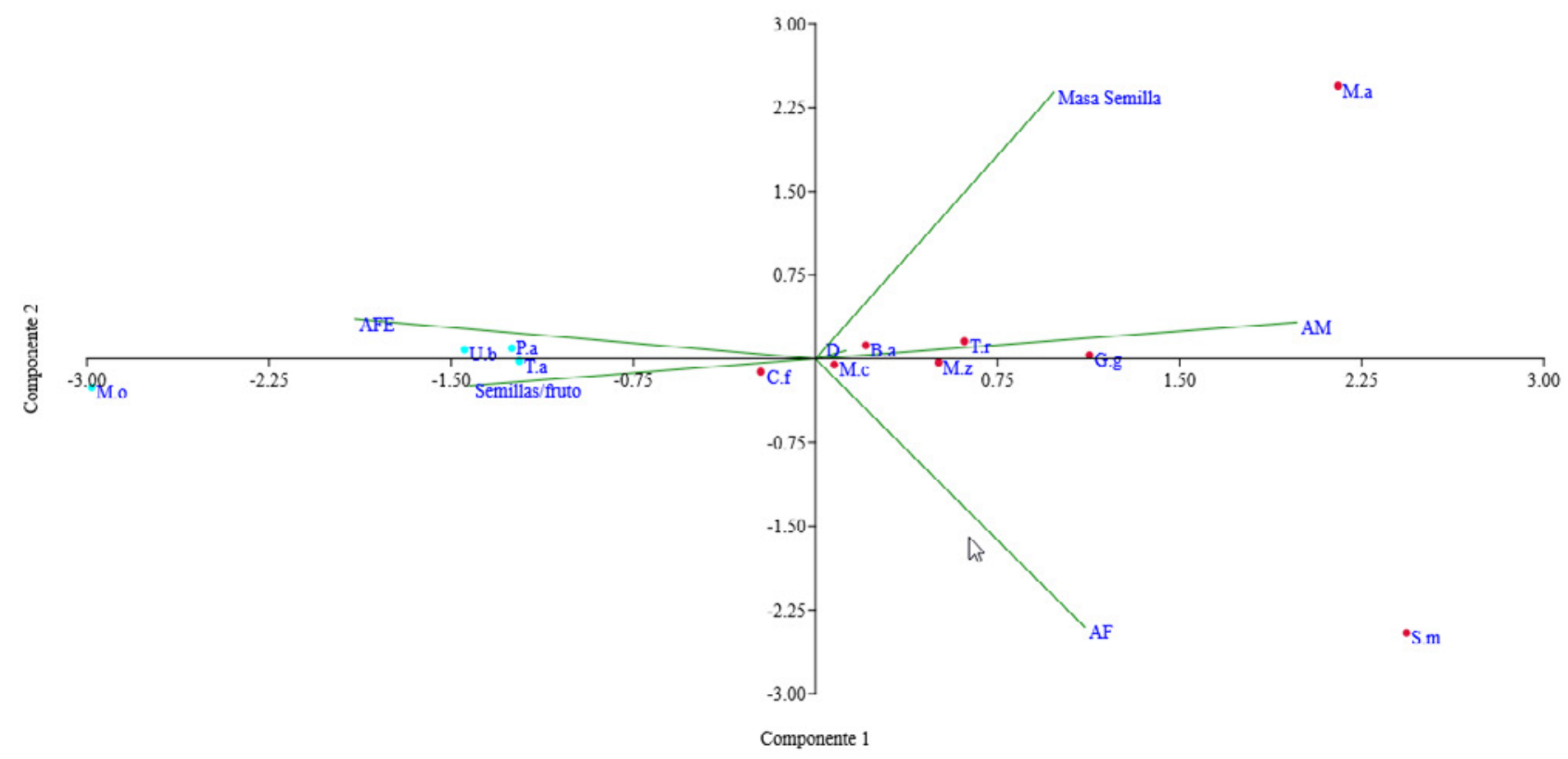

Figura 2. Análisis de componentes PCA de los grupos funcionales de la parcela.

B.a = Brossimum alicastrum, C.f = Capparidastrum frondosum, G.g = Guarea guidonia, M.a = Mammea americana, M.c = Manilkara chicle, M.o = Martinella obovata, M.z = Manilkara zapota, P.a = Piper amalago, S.m = Sabal mauritiiformis, T.a = Tabernaemontana amygdalifolia, T.r = Trophis racemosa, U.b = Urera baccifera. 
proporción de área foliar específica, y por habitar zonas abiertas o de sotobosque.

\section{Diversidad filogenética}

Al realizar el análisis de la diversidad filogenética de la comunidad vegetal que alberga el bs-T del JBGP (todos los cuadrantes $\mathrm{n}=25$ ), el valor de PSV encontrado (0.67) indicó que las especies registradas están poco relacionadas filogenéticamente entre sí. Por su parte, el valor de PSR mostró una riqueza filogenética de 8.37 , el PSE presentó un valor medio de 0.58 y el PSC un valor bajo (0.38).

\section{DISCUSIÓN}

Para empezar, la riqueza de especies presentes en el bs-T del JBGP (62) se encuentra dentro del rango reportado (55 a 155 especies) para otros bs-T en el Caribe colombiano (Marulanda et al., 2003; Mendoza-C., 1999). El índice de diversidad de Shannon $\mathrm{H}^{\prime}$ refleja valores cercanos a 3, lo que indica una alta diversidad, mientras que la exponencial de Shannon $\mathrm{e}^{\left(\mathrm{H}^{\prime}\right)}$ muestra que la cantidad efectiva de especies es mucho menor a la riqueza de la parcela. A su vez, el índice de diversidad de Simpson (1-D) sugiere una baja dominancia y una alta diversidad, aunque el resultado del valor del índice de diversidad de Simpson difiere de lo observado dada la dominancia de dos especies en la parcela. Tal es el caso de la alta abundancia de individuos de Moraceae: Brosimum alicastrum y Trophis racemosa, especies perennifolias y comunes en otros bs-T del Caribe colombiano (Herazo Vitola et al., 2017). Al respecto, se encontró que casi la totalidad de sus individuos son árboles juveniles que crecen en el sotobosque, con Brosimum alicastrum y Trophis racemosa exponiendo en el $92 \%$ y el $70 \%$ de sus individuos, respectivamente, un DAP menor de $10 \mathrm{~cm}$. Por otra parte, sólo el $6 \%$ de los individuos de Brosimum alicastrum y el $24 \%$ de Trophis racemosa alcanzan el dosel.
En cuanto a la riqueza de especies, la familia Sapindaceae presentó la mayor cantidad, con cuatro: los árboles Sapindus saponaria, Melicoccus bijugatus y Melicoccus oliviformis, y la liana Paullinia sp. Si bien esta familia es una de las dominantes en los bs-T (Gentry, 1995), en el bosque del JBGP está representada por unos pocos individuos aislados (6 en total). Así pues, los valores de abundancia de lianas ( $11 \%$ del total de individuos registrados) son similares a los de otros bs-T del Caribe colombiano, los cuales se encuentran entre el $5 \%$ y el 20 $\%$ del total de individuos (Marulanda et al., 2003; Polania Cleves, 2019); también se encontró este mismo patrón de diversidad para Bignoniaceae, que la señala como la familia más representativa entre las lianas (Gentry, 1995).

Ahora bien, pese a que las familias registradas para el bosque del JBGP hacen parte de la composición característica de los bs-T, uno de los hallazgos más interesantes fue la poca representatividad de Fabaceae, de la cual se registraron tres especies (dos lianas y un árbol), con un total de siete individuos y un IVI acumulado de $6 \%$, lo que contrasta con la dominancia constante de esta familia en el estrato arbóreo del bs-T en el neotrópico (Gentry, 1995; Mendoza-C., 1999; Pizano et al., 2014; Vargas et al., 2015). Es necesario señalar que un posible factor determinante en la composición del bosque del JBGP es la dispersión de semillas por vertebrados. Esto se hace notorio en el predominio de especies productoras de frutos carnosos en todos los estratos del bosque y concuerda con la abundancia de fauna silvestre observada en la zona durante el censo (Janzen, 1988). En este sentido, la poca representatividad de Fabaceae puede ser consecuencia de la ausencia de dispersores efectivos de semillas. En el caso de Alouatta seniculus y Saguinus oedipus (mono aullador y mono tití cabeciblanco, especies que habitan en el JBGP), se ha reportado que el principal recurso alimenticio extraído de Fabaceae es la savia y las hojas (Ávila Molina y Padilla Rivera, 2005; García Castillo, 2016). Así mismo, se ha constatado la ausencia de lluvia de semillas de esta familia en fragmentos de bs- $T$ del Caribe colombiano, en donde los principales 
dispersores son aves y murciélagos (León Barbosa, 2010). Por otro lado, la presencia de individuos de especies cultivadas de uso ornamental o frutal dentro de la parcela, como Manilkara zapota, Mammea americana, Roystonea regia y Adonidia merrillii, puede deberse a la dispersión de sus frutos por parte de los mamíferos en el área en que se alimentan de ellas (el argumento anterior proviene de observaciones en campo durante los censos).

Respecto al valor obtenido de biomasa aérea total, la parcela JBGP (con 61.8 t.ha $^{-1}$ ) está dentro del rango de los valores obtenidos $\left(35\right.$ t.ha $^{-1}$ a 88 t.ha $^{-1}$ ) para otras parcelas de bs-T localizadas en fragmentos de estado intermedio a tardío de sucesión en Colombia (Polania Cleves, 2019; Torres et al., 2012). En este sentido, al analizar la biomasa por especies, se encontró que Guarea guidonia aportó la mayor biomasa aérea, lo que está relacionado con el gran tamaño de los individuos de esta especie, pues presenta menos de un cuarto de la cantidad de individuos de Trophis racemosa o Brosimum alicastrum (64 individuos contra 270 individuos). Así pues, el $50 \%$ de los individuos de Guarea guidonia alcanzan el dosel, el $25 \%$ son árboles emergentes (altura mayor a $14 \mathrm{~m}$, con un máximo de $24 \mathrm{~m}$ ) y el $20 \%$ de los tallos presentan un DAP mayor a $30 \mathrm{~cm}$.

Sobre los índices de diversidad funcional, estos sugieren que para los rasgos escogidos no hay alta divergencia entre las especies y, por ende, tampoco a nivel de la comunidad. El valor del índice rRao indica, por tanto, que la comunidad es menos de la mitad de diversa de lo que podría ser en cuanto a caracteres funcionales (42\%), lo que soporta la idea de homogeneidad en ella. El índice FEve muestra también que la comunidad es bastante parecida, y que solo aproximadamente el $30 \%$ de las especies cuentan con un rasgo diferente. En consecuencia, los dos tipos funcionales encontrados se caracterizan principalmente por tres rasgos: la altura máxima y la densidad de la madera, relacionadas con la forma de crecimiento, y el área foliar específica, relacionada con el costo de producción de la superficie foliar. Aun así, si bien los valores de los índices de diversidad funcional muestran que no existen grandes diferencias entre los tipos funcionales, es notable la agrupación de las especies arbóreas con la palma Sabal mauritiiformis en un ensamblaje funcional con mayor densidad de madera y baja área foliar específica.

Cabe resaltar que las especies seleccionadas para el análisis funcional se caracterizaron por presentar follaje perenne de hojas simples, dispersión por animales y fructificación no estacional, características que contrastan con los patrones generales reportados para los bs-T, donde son más comunes las especies caducifolias, de hojas compuestas, dispersión anemócora y, en general, una fenología determinada por la estacionalidad en las Iluvias (Murphy y Lugo, 1986; Pizano et al., 2014). En relación con esto, existen estudios que muestran que al aumentar la disponibilidad de agua los patrones fenológicos tienden a ser menos estacionales (Borchert, 1994). Lo anterior sugiere que para las especies del bosque del JBGP la abundancia de acuíferos y de corrientes de agua en la zona puede ser un factor importante que afecta la fenología y la estructura observadas. Además, adicional a la presencia de acuíferos, el nivel de degradación actual que se observa en zonas aledañas también puede ser un factor a evaluar a futuro con el objetivo de entender su influencia en la composición y estructura observadas (Janzen, 1988; Miles et al., 2006).

Finalmente, acerca de la diversidad filogenética existe un bajo agrupamiento en las especies del bosque que difiere de los dos grupos encontrados con base a los rasgos funcionales. Por un lado, el PSV indica que las especies no están muy relacionadas entre sí, lo cual se ve reflejado en la dispersión de las familias de las especies a lo largo del árbol filogenético de las Angiospermas. Por otro lado, el PSR sugiere que hay un aproximado de ocho grupos filogenéticos que comparten características similares. De ahí que al añadir el ponderador de abundancia al PSV se obtenga el valor del PSE, lo que sugiere una alta variabilidad en la abundancia de las especies. Esto se relaciona con la dominancia antes mencionada de Brosimum 
alicastrum y Trophis racemosa. Por su parte, el PSC sugiere un agrupamiento alto en las puntas de las ramas del dendrograma, indicando así que la mayoría de las familias examinadas se encuentran en los mismos grupos y que en algunos casos se registraron especies muy cercanas dentro de un mismo género o en géneros hermanos.

\section{CONCLUSIONES}

Teniendo en cuenta su composición y estructura, el bs-T que alberga el JBGP se encuentra en un estado de sucesión secundario intermedio a tardío. La baja representatividad de Fabaceae en este remanente es inusual en el bs-T, lo cual puede deberse a su aislamiento frente a otros relictos de bs-T, limitando así la dispersión de semillas. A su vez, su papel como refugio de fauna en la zona parece reforzar el dominio de especies dispersadas por vertebrados y su localización en un sitio con alta disponibilidad de agua promueve el asentamiento de especies no estacionales. Pese esto, conserva muchas características del bs- $\mathrm{T}$.

En general, la vegetación del bosque del JBGP parece estar más determinada por su diversidad funcional debido a la diferencia entre los grupos funcionales encontrados y a la baja dominancia observada en cuanto a composición -que se corresponde con la diversidad filogenética-. Es necesario, entonces, continuar con el estudio de otros fragmentos aislados en esta región, ya que estos aportan información sobre el impacto de degradación actual del bs-T caribeño. Los monitoreos y estudios sobre su dinámica ecológica permitirán futuras investigaciones sobre las relaciones de competencia y el establecimiento de nuevas especies en la zona.

\section{AGRADECIMIENTOS}

Los autores agradecen a la Fundación para la Promoción de la Investigación y la Tecnología del
Banco de la República (FPIT) y al JBGP por la financiación recibida para este proyecto. También a todo el personal del herbario y el vivero del JBGP por su ayuda y apoyo. Adicionalmente, a los dos evaluadores anónimos que con sus aportes enriquecieron el contenido del presente artículo.

\section{CONTRIBUCIONES POR AUTOR}

V. L.L., J. T.G. y S. M. idearon la investigación y escribieron el proyecto y la metodología. J. T.G. y O. E.H. realizaron la toma de datos en campo y el análisis preliminar de los datos. V. L.L., J. T.G. y O. E.H. escribieron la versión inicial del artículo. Todos los autores realizaron la edición final del texto y contribuyeron en su discusión y conclusiones.

\section{CONFLICTO DE INTERESES}

Los autores declaran no tener conflicto de intereses.

\section{REFERENCIAS}

Abràmoff, M. D., Magalhães, P. J. y Ram, S. J. (2004). Image Processing with ImageJ. Biophotonics International, 11(7), 36-42.

Álvarez, E., Benítez, D., Velásquez, C. y Cogollo, Á. (2013). Densidad básica del fuste de árboles del bosque seco en la costa Caribe de Colombia. Intropica, 8(1), 17-28.

Álvarez, E., Mendoza, I., Pacheco, M., Restrepo, Z., Benítez, D., Gutiérrez, T. D., Ramírez, O. C., Dib, J. C., Roldán, A., Carbono, E., Zarza, E., Velásquez, L. A., Serna, M., Velásquez, C., Álvarez, Y., Jiménez, O., Martínez, M., Idárraga, A. y Gómez, F. (2012). ¿Por qué implementar estudios de largo plazo en el bosque seco del Caribe colombiano? Intropica, 7(1), 97-113.

Ávila Molina, F. y Padilla Rivera, O. (2005). Variación estacional del uso de recursos alimenticios del mono aullador Alouatta seniculus (L. 1766) en un 
fragmento de bosque seco tropical (bs-T) en el norte del departamento del Atlántico, Colombia [Trabajo de grado, Universidad del Atlántico].

https://www.researchgate.net/profile/Orlando-Rivera-6/publication/330114331_Alouatta_seniculus-BsT-edited/links/5c2e28c8458515a4c70a5339/ Alouatta-seniculus-BsT-edited.pdf

Borchert, R. (1994). Soil and stem water storage determine phenology and distribution of tropical dry forest trees. Ecology, 75(5), 1437-1449.

https://doi.org/10.2307/1937467

Botero Pareja, C. E., Guardo Castaño, L. G., Nieto Parra, G. y Castaño Rodríguez, G. F. (2008). Caracterización hidrológica de la cuenca del arroyo Matute como el elemento para considerar un posible ordenamiento territorial [Tesis de Maestría, Pontificia Universidad Javeriana]. Repositorio institucional de la Universidad Tecnológica de Bolívar (UTB).

https://repositorio.utb.edu.co/handle/20.500 $.12585 / 1501$

Casanoves, F., Pla, L., Di Rienzo, J. A. y Díaz, S. (2010). FDiversity: a software package for the integrated analysis of functional diversity. Methods in Ecology and Evolution, 2(3), 233-237.

https://doi.org/10.1111/j.2041-210X.2010.00082.x

Casanoves, F., Pla, L. y Di Rienzo, J. A. (2011). Valoración y análisis de la diversidad funcional y su relación con los servicios ecosistémicos. Centro Agronómico Tropical de Investigación y Enseñanza (CATIE).

http://repositorio.bibliotecaorton.catie.ac.cr/ handle/11554/8190

Cedeño, E., Viteri, K. y Costa, A. (2010). Estudio del comportamiento de la pulpa congelada y del aceite de semillas obtenido de dos variedades diferentes de mamey Colocarpum mammosum (mamey colorado) y Mammea americana (mamey Cartagena) [Trabajo de grado, Escuela Superior Politécnica del Litoral]. Repositorio institucional de la Escuela Superior Politécnica del Litoral.

h t tp://www.dspace.espol.edu.ec/ handle/123456789/8848

Chave, J., Réjou-Méchain, M., Búrquez, A., Chidumayo, E., Colgan, M. S., Delitti, W. B. C., Duque, A.,
Eid, T., Fearnside, P. M., Goodman, R. C., Henry, M., Martínez-Yrízar, A., Mugasha, W. A., Muller-Landau, H. C., Mencuccini, M., Nelson, B. W., Ngomanda, A., Nogueira, E. M., Ortiz-Malavassi, E.,...y Vieilledent, G. (2014). Improved allometric models to estimate the aboveground biomass of tropical trees. Global Change Biology, 20(10), 3177-3190.

https://doi.org/10.1111/gcb.12629

DRYFLOR, Bnada-R, K., Delgado-Salinas, A., Dexter, K. G., Linares-Palomino, R., Oliveira-Filho, A., Prado, D., Pullan, M., Quintana, C., Riina, R., Rodríguez, G. M., Weintritt, J., Acevedo-Rodríguez, P., Adarve, J., Álvarez, E., Aranguren, A., Arteaga, J. C., Aymard, G., Castaño, A.,...Pennington, R. T. (2016). Plant diversity patterns in neotropical dry forests and their conservation implications. Science, 353(6306), 1383-1387.

https://doi.org/10.1126/science.aaf5080

Faicán Ortiz, K. V. e Intriago Méndez, N. L. (2019). Propuesta de aprovechamiento de la semilla del Chicozapote (Manilkara zapota) en la culinaria [Trabajo de grado, Universidad de Guayaquil]. Repositorio institucional de la Universidad de Guayaquil. http://repositorio.ug.edu.ec/handle/redug/47007

FAO [Food and Agriculture Organization of the United Nations]. (2019). Trees, forests and land use in drylands: the first global assessment - full report (FAO Forestry Paper No.184).

http://www.fao.org/3/ca7148en/ca7148en.pdf

Francis, J. K. y Rodríguez, A. (1993). Seeds of Puerto Rican trees and shrubs: second installment (Research Note SO-374). U.S. Department of Agriculture/ Forest Service/ Southern Forest Experiment Station. https://www.fs.usda.gov/treesearch/pubs/1686

García Castillo, E. F. (2016). Preferencias alimenticias y dispersión de semillas por grupos silvestres del Tití Cabeciblanco (Saguinus oedipus, primates) en un bosque seco tropical [Tesis de Maestría, Universidad Nacional de Colombia]. Repositorio institucional de la Universidad Nacional de Colombia. https://repositorio.unal.edu.co/handle/unal/55545

Gentry, A. H. (1995). Diversity and floristic composition of neotropical dry forests. En S. H. Bullock, H. A. 
Mooney y E. Medina (Eds.), Seasonally dry tropical forests ( $1^{\text {a }}$ ed) (pp. 146-194). Cambridge University Press.

https://doi.org/10.1017/CBO9780511753398.007

Gerhardt, K. (1993). Tree seedling development in tropical dry abandoned pasture and secondary forest in Costa Rica. Journal of Vegetation Science, 4(1), 95102. https://doi.org/10.2307/3235736

González-M, R., García, H., Isaacs, P., Cuadros, H., López-Camacho, R., Rodríguez, N., Pérez, K., Mijares, F., Castaño-Naranjo, A., Jurado, R., Idárraga-Piedrahíta, A., Rojas, A., Vergara, H. y Pizano, C. (2018). Disentangling the environmental heterogeneity, floristic distinctiveness and current threats of tropical dry forests in Colombia. Environmental Research Letters, 13(4), 045007.

Hammer, Ø., Harper, D. A. T. y Ryan, P. D. (2001). PAST: Paleontological Statistics Software Package for Education and Data Analysis (Versión 4.0.2) [Software de computador].

https://palaeo-electronica.org/2001_1/past/issue1_01.htm

Helmus, M. R., Bland, T. J., Williams, C. K. e Ives, A. R. (2007). Phylogenetic measures of biodiversity. The American Naturalist, 169(3), E68-E83.

https://doi.org/10.1086/511334

Herazo Vitola, F. Y., Mercado Gómez, J. y Mendoza Cifuentes, H. (2017). Estructura y composición florística del bosque seco tropical en los Montes de María (Sucre - Colombia). Ciencia en Desarrollo, $8(1), 71-82$.

https://doi.org/10.19053/01217488. v8.n1.2017.5912

Janzen, D. H. (1988). Management of habitat fragments in a tropical dry forest: growth. Annals of the Missouri Botanical Garden, 75(1), 105-116. https://doi. org/10.2307/2399468

Jost, L. (2007). Partitioning diversity into independent alpha and beta components. Ecology, 88(10), 2427 2439. https://doi.org/10.1890/06-1736.1

León Barbosa, E. (2010). Lluvia de semillas efectuada por aves y murciélagos hacia pastizales asociados a un fragmento de bosque seco tropical (Córdoba-Colombia) [Trabajo de grado, Pontificia
Universidad Javeriana]. Repositorio institucional de la Pontificia Universidad Javeriana.

https://repository.javeriana.edu.co/bitstream/handle/10554/8503/tesis 461 . pdf? sequence $=1$ \&isAllowed $=y$

Londoño-Lemos, V., Escobar-Hadechini, O., Tapasco-García, J. y Madriñán, S. (2020). Composición florística y estructura vegetal de una parcela permanente en un relicto de bosque seco tropical en el Caribe colombiano (Versión 1.1.) [Conjunto de datos]. SiB Colombia.

https://doi.org/10.15472/kxbwev

Maia, V. A., de Souza, C. R., de Aguiar-Campos, N., Fagundes, N. C. A., Santos, A. B. M., de Paula, G. G. P., Santos, P. F., Silva, W. B., Menino, G. C. O. y dos Santos, R. M. (2020). Interactions between climate and soil shape tree community assembly and above-ground woody biomass of tropical dry forests. Forest Ecology and Management, 474, 118348.

https://doi.org/10.1016/j.foreco.2020.118348

Marulanda, L. O., Uribe, A., Velásquez, P., Montoya, M. Á., Idárraga, Á., López, M. C. y López, J. M. (2003). Estructura y composición de la vegetación de un fragmento de bosque seco en San Sebastián, Magdalena (Colombia). I. Composición de plantas vasculares. Actualidades Biológicas, 25(78), 17-30.

Mendoza-C., H. (1999). Estructura y riqueza florística del bosque seco tropical en la región Caribe y el Valle del río Magdalena, Colombia. Caldasia, 21(1), 70-94.

Miles, L., Newton, A. C., DeFries, R. S., Ravilious, C., May, I., Blyth, S., Kapos V. y Gordon, J. E. (2006). A global overview of the conservation status of tropical dry forests. Journal of Biogeography, 33(3), 491-505. https://doi.org/10.1111/j.1365-2699.2005.01424.x

Murphy, P. G. y Lugo, A. E. (1986). Ecology of tropical dry forest. Annual Review of Ecology and Systematics, 17(1), 67-88.

https://doi.org/10.1146/annurev. es.17.110186.000435

Pennington, R. T., Lavin, M. y Oliveira-Filho, A. (2009). Woody plant diversity, evolution, and ecology in the tropics: perspectives from seasonally dry 
tropical forests. Annual Review of Ecology, Evolution, and Systematics, 40, 437-457.

https://doi.org/10.1146/annurev.ecolsys.110308.120327

Pérez-Harguindeguy, N., Díaz, S., Garnier, E., Lavorel, S., Poorter, H., Jaureguiberry, P., Bret-Harte, M. S., Cornwell, W. K., Craine, J. M., Gurvich, D. E., Urcelay, C., Veneklaas, E. J., Reich, P. B., Poorter, L., Wright, I. J., Ray, P., Enrico, L., Pausas, J. G., de Vos, A. C.,... y Cornelissen, J. H. C. (2013). New handbook for standardised measurement of plant functional traits worldwide. Australian Journal of Botany, 61(3), 167-234.

https://doi.org/10.1071/BT12225_CO

Pizano, C., Cabrera, M. y García, H. (2014). Bosque seco tropical en Colombia; generalidades y contexto. En C. Pizano y H. García (Eds.), El bosque seco tropical en Colombia ( $1^{\text {a }}$ ed.) (pp. 37-47). Instituto de Investigación de Recursos Biológicos Alexander von Humboldt $(\mathrm{IAvH})$.

http://repository.humboldt.org.co/handle/20.500 $.11761 / 9333$

Pizano, C., González-M., R., López, R., Jurado, R. D., Cuadros, H., Castaño-Naranjo, A., Rojas, A., Pérez, K., Vergara-Varela, H., Idárraga, Á., Isaacs, P. y García, H. (2016). El bosque seco tropical en Colombia. En M. F. Gómez, L. A. Moreno, G. I. Andrade y C. Rueda (Eds.), Biodiversidad 2015. Estado y tendencias de la biodiversidad continental de Colombia ( $1^{\mathrm{a}}$ ed) (pp. 22). Instituto de Investigación de Recursos Biológicos Alexander von Humboldt (IAvH).

http://www.humboldt.org.co/es/estado-de -los-recursos-naturales/item/898-bio2015

Polania Cleves, K. A. (2019). Biomasa aérea en estados sucesionales del bosque seco tropical en el norte del Tolima, Colombia [Trabajo de grado, Universidad Distrital Francisco José de Caldas]. Repositorio institucional de la Universidad Distrital Francisco José de Caldas.

https://repository.udistrital.edu.co/handle/11 349/15488

Putz, F. E. (1990). Liana stem diameter growth and mortality rates on Barro Colorado Island, Panama. Biotropica, 22(1), 103-105.

https://doi.org/10.2307/2388725
Resolución 000571 de 2015 [Ministerio de Ambiente y Desarrollo Sostenible]. Por la cual se otorga una Licencia Ambiental y se dictan otras disposiciones. 09 de febrero de 2015.

Royal Botanic Gardens Kew. (2021). Seed Information Database (SID) (Versión 7.1.) [Conjunto de datos]. http://data.kew.org/sid/

Ruiz Linares, J. y Fandiño Orozco, M. C. (2009). Estado del bosque seco tropical e importancia relativa de su flora leñosa, Islas de la Vieja Providencia y Santa Catalina, Colombia, Caribe suroccidental. Rev. Acad. Colomb. Cienc., 33(126), 5-15.

Sánchez-Azofeifa, A., Kalacska, M. E., Quesada, M., Stoner, K. E., Lobo, J. A. y Arroyo-Mora, P. (2003). Tropical Dry Climates. En M. Schwartz (Ed.), Phenology: an integrative environmental science $\left(1^{\mathrm{a}} \mathrm{ed}\right)$ (pp. 121-137).

https://doi.org/10.1007/978-94-007-0632-3_9

Schnitzer, S. A., DeWalt, S. J. y Chave, J. (2006). Censusing and measuring lianas: a quantitative comparison of the common methods. Biotropica, 38(5), 581-591. https://doi.org/10.1111/j.1744-7429.2006.00187.x

Torres, A. M., Adarve, J. B., Cárdenas, M., Vargas, J. A., Londoño, V., Rivera, K., Home, J., Duque, $O$. L. y González, Á. M. (2012). Dinámica sucesional de un fragmento de bosque seco tropical del Valle del Cauca, Colombia. Biota Colombiana, 13(2), 66-85.

Vallejo-Joyas, M. I., Londoño-Vega, A. C., López-Camacho, R., Galeano, G., Álvarez-Dávila, E. y DeviaÁlvarez, W. (2005). Establecimiento de parcelas permanentes en bosques de Colombia. Volumen I. Instituto de Investigación de Recursos Biológicos Alexander von Humboldt.

http://repository.humboldt.org.co/handle/20.500 $.11761 / 34195$

Vargas, G., Werden, L. K. y Powers, J. S. (2015). Explaining legume success in tropical dry forest based on seed germination niches: a new hypothesys. Biotropica, 47(3), 277-280.

https://doi.org/10.1111/btp.12210

Villéger, S., Ramos Miranda, J., Flores Hernández, D. y Mouillot, D. (2010). Contrasting changes in taxonomic vs. functional diversity of tropical fish 
communities after habitat degradation. Ecological applications, 20(6), 1512-1522.

https://doi.org/10.1890/09-1310.1

Webb, C. O. y Donoghue, M. J. (2005). Phylomatic: tree assembly for applied phylogenetics. Molecular Ecology Notes, 5(1), 181-183.

https://doi.org/10.1111/j.1471-8286.2004.00829.x
Zanne, A. E., Lopez-Gonzalez, G., Coomes, D. A., Ilic, J., Jansen, S., Lewis, S. L., Miller, R. B., Swenson, N. G., Wiemann, M. C., y Chave, J. (2009). Global Wood Density Database [Conjunto de datos]. DRYAD. https://doi.org/10.5061/dryad.234 


\section{ANEXOS}

Anexo 1. Especies registradas en la parcela de 1 ha perteneciente al JBGP. Se presentan los datos de la sumatoria total de área basal $\left(\mathrm{m}^{2}\right)$, biomasa (t.ha-1), número de tallos por especie e índice de valor de importancia (IVI) porcentual (el valor de IVI \% corresponde a la sumatoria de tres porcentajes, por lo que su máximo valor es 300)

\begin{tabular}{|c|c|c|c|c|c|}
\hline Familia & Especie & $\begin{array}{l}\text { Área basal } \\
\left(\mathrm{m}^{2}\right)\end{array}$ & $\begin{array}{c}\text { Biomasa } \\
(\text { t.ha-1) }\end{array}$ & Tallos & IVI (\%) \\
\hline Achatocarpaceae & Achatocarpus nigricans & 0.610 & 1.258 & 69 & 23 \\
\hline \multirow{2}{*}{ Anacardiaceae } & Anacardium excelsum & 0.008 & $0-007$ & 4 & 4 \\
\hline & Spondias radlkoferi & 0.126 & 0.339 & 1 & 2 \\
\hline \multirow{3}{*}{ Apocynaceae } & Rauvolfia viridis & 0.084 & 0.198 & 6 & 3 \\
\hline & Tabernaemontana amygdalifolia & 0.083 & 0.095 & 66 & 29 \\
\hline & Tabernaemontana cymosa & 0.143 & 0.264 & 5 & 3 \\
\hline \multirow{3}{*}{ Arecaceae } & Adonidia merrillii & 0.002 & 0.001 & 1 & 1 \\
\hline & Roystonea regia & 0.192 & 0.351 & 3 & 3 \\
\hline & Sabal mauritiiformis & 2.003 & 4.780 & 52 & 42 \\
\hline Asteraceae & Mikania globifera & 0.001 & 0.014 & 2 & 1 \\
\hline Bignoniaceae & Martinella obovata & 0.179 & 2.515 & 117 & 42 \\
\hline \multirow{2}{*}{ Boraginaceae } & Cordia & 0.002 & 0.001 & 1 & 1 \\
\hline & Tournefortia angustiflora & 0.020 & 0.575 & 2 & 2 \\
\hline Calophyllaceae & Mammea americana & 0.971 & 3.240 & 133 & 52 \\
\hline \multirow{2}{*}{ Capparaceae } & Capparidastrum frondosum & 0.186 & 0.341 & 175 & 70 \\
\hline & Quadrella indica & 0.020 & 0.039 & 7 & 4 \\
\hline Caricaceae & Carica papaya & 0.012 & 0.005 & 6 & 4 \\
\hline Combretaceae & Combretum decandrum & 0.012 & 0.162 & 8 & 5 \\
\hline \multirow{2}{*}{ Euphorbiaceae } & Acalypha macrostachya & 0.008 & 0.010 & 7 & 1 \\
\hline & Hura crepitans & 0.003 & 0.001 & 2 & 2 \\
\hline \multirow{3}{*}{ Fabaceae } & Gliricidia sepium & 0.083 & 0.267 & 1 & 2 \\
\hline & Pithecellobium roseum & 0.005 & 0.061 & 3 & 1 \\
\hline & Senegalia tenuifolia & 0.013 & 0.165 & 12 & 3 \\
\hline \multirow{3}{*}{ Lamiaceae } & Aegiphila laeta & 0.037 & 0.724 & 9 & 5 \\
\hline & Vitex & 0.009 & 0.021 & 1 & 1 \\
\hline & Lamiaceae & 0.012 & 0.032 & 10 & 2 \\
\hline Lauraceae & Nectandra turbacensis & 0.702 & 2.198 & 22 & 10 \\
\hline Lecythidaceae & Gustavia superba & 0.002 & 0.003 & 1 & 1 \\
\hline Malpighiaceae & Malpighia emarginata & 0.020 & 0.033 & 12 & 6 \\
\hline \multirow{2}{*}{ Malvaceae } & Cavanillesia platanifolia & 0.005 & 0.004 & 3 & 3 \\
\hline & Sterculia apetala & 1.344 & 4.022 & 3 & 10 \\
\hline \multirow{3}{*}{ Meliaceae } & Cedrela odorata & 0.986 & 2.634 & 4 & 9 \\
\hline & Guarea guidonia & 4.362 & 14.111 & 93 & 60 \\
\hline & Trichilia martiana & 0.726 & 2.266 & 31 & 18 \\
\hline \multirow{3}{*}{ Moraceae } & Brosimum alicastrum & 0.766 & 1.861 & 274 & 94 \\
\hline & Ficus maxima & 2.616 & 6.895 & 19 & 28 \\
\hline & Trophis racemosa & 2.486 & 6.464 & 274 & 109 \\
\hline \multirow{3}{*}{ Nyctaginaceae } & Neea divaricata & 0.020 & 0.027 & 3 & 1 \\
\hline & Neea virens & 0.003 & 0.007 & 1 & 2 \\
\hline & Pisonia aculeata & 0.063 & 1.389 & 13 & 1 \\
\hline Petiveriaceae & Trichostigma octandrum & 0.037 & 0.608 & 15 & 4 \\
\hline
\end{tabular}




\begin{tabular}{|c|c|c|c|c|c|}
\hline Familia & Especie & $\begin{array}{c}\text { Área basal } \\
\left(\mathrm{m}^{2}\right)\end{array}$ & $\begin{array}{c}\text { Biomasa } \\
\left(\mathrm{t} . \mathrm{ha}^{-1}\right)\end{array}$ & Tallos & IVI (\%) \\
\hline \multirow{2}{*}{ Piperaceae } & Piper amalago & 0.128 & 0.125 & 139 & 9 \\
\hline & Piper reticulatum & 0.027 & 0.019 & 20 & 34 \\
\hline \multirow{2}{*}{ Polygonaceae } & Coccoloba obovata & 0.021 & 0.027 & 7 & 3 \\
\hline & Triplaris americana & 0.002 & 0.002 & 1 & 2 \\
\hline Primulaceae & Primulaceae & 0.001 & 0.001 & 1 & 1 \\
\hline \multirow{2}{*}{ Rubiaceae } & Pittoniotis trichantha & 0.250 & 0.759 & 3 & 3 \\
\hline & Psychotria carthagenensis & 0.001 & 0.001 & 1 & 1 \\
\hline \multirow{2}{*}{ Rutaceae } & Amyris elemifera & 0.000 & 0.001 & 1 & 4 \\
\hline & Zanthoxylum & 0.021 & 0.081 & 1 & 1 \\
\hline Salicaceae & Casearia aculeata & 0.004 & 0.007 & 4 & 4 \\
\hline \multirow{4}{*}{ Sapindaceae } & Melicoccus bijugatus & 0.001 & 0.001 & 1 & 1 \\
\hline & Melicoccus oliviformis & 0.007 & 0.024 & 2 & 2 \\
\hline & Paullinia & 0.001 & 0.008 & 2 & 2 \\
\hline & Sapindus saponaria & 0.018 & 0.044 & 1 & 1 \\
\hline \multirow{2}{*}{ Sapotaceae } & Manilkara chicle & 0.047 & 0.165 & 32 & 21 \\
\hline & Manilkara zapota & 0.307 & 0.493 & 87 & 37 \\
\hline \multirow[t]{2}{*}{ Simaroubaceae } & Simarouba amara & 0.051 & 0.067 & 5 & 4 \\
\hline & Cecropia peltata & 0.468 & 0.925 & 16 & 13 \\
\hline \multirow[t]{2}{*}{ Urticaceae } & Urera baccifera & 0.446 & 0.155 & 170 & 20 \\
\hline & Urera caracasana & 0.078 & $0 . .043$ & 41 & 5 \\
\hline Vitaceae & Vitis tiliifolia & 0.022 & 0.291 & 17 & 8 \\
\hline
\end{tabular}

